Mon. Not. R. Astron. Soc. 000, 000-000 (0000) Printed 16 December $2018 \quad$ (MN LATEX style file v2.2)

\title{
X-rays from the Type II quasar in the hyperluminous infrared galaxy IRAS F15307+3252
}

\author{
K. Iwasawa ${ }^{1}$, C.S. Crawford ${ }^{1}$, A.C. Fabian ${ }^{1}$ and R.J. Wilman ${ }^{2}$ \\ ${ }^{1}$ Institute of Astronomy, Madingley Road, Cambridge CB3 OHA \\ ${ }^{2}$ Department of Physics, University of Durham, South Road, Durham DH1 3LE
}

\begin{abstract}
We report the detection of X-ray emission from the hyperluminous infrared galaxy IRAS F15307+3252 at $z=0.93$ and its properties obtained from XMM-Newton observations. Although the X-ray emission is very faint and the data are noisy, a prominent line-like feature in the observed 3-4 keV range is inferred from both photometric and spectroscopic techniques. It indicates an X-ray spectrum dominated by $6.4 \mathrm{keV}$ Fe $\mathrm{K} \alpha$ emission and the presence of a Compton-thick AGN. Our estimate of the luminosity of the illuminating source $\left(L_{2-10 \mathrm{keV}} \geqslant 1 \times 10^{45} \mathrm{erg} \mathrm{s}^{-1}\right)$, required to produce the observed $\mathrm{Fe} \mathrm{K} \alpha$ emission in reflection from cold matter, means that the hidden quasar nucleus accounts for a significant fraction of the large bolometric luminosity. The soft X-ray emission below $2 \mathrm{keV}$ is found to be spatially extended and probably of a separate origin. The temperature and bolometric luminosity ( $k T \simeq 2 \mathrm{keV}$ and $L_{\mathrm{bol}}^{\mathrm{CL}} \simeq 1 \times 10^{44} \mathrm{erg} \mathrm{s}^{-1}$ ) obtained from a thermal spectrum place this X-ray source on the $L-T_{\mathrm{X}}$ relation of galaxy clusters. The possible association with a galaxy cluster can be added to the list of remarkable similarities between IRAS F15307+3252 and another hyperluminous infrared galaxy IRAS $09104+4109(z=0.44)$, both of which have bolometric luminosities dominated by hidden quasar nuclei. Our result on IRAS F15307+3252 illustrates how difficult it is to detect Compton thick Type II quasars at $z=1$, particularly if their bolometric outputs do not rival the hyperluminous population.
\end{abstract}

Key words: Galaxies: individual: IRAS F15307+3252 — X-rays: galaxies — X-rays: galaxies: clusters — infrared: galaxies

\section{INTRODUCTION}

The class of hyperluminous infrared galaxies, whose bolometric luminosities exceed $10^{13} L_{\odot}$, have been found through far-infrared and submillimetre surveys by IRAS and SCUBA (e.g., RowanRobinson 2000), and more are expected from the Spitzer Space Telescope. Some of them may be massive galaxies in formation at high redshift and, given the suggested link between galaxy spheroids and central black holes in them (e.g., Tremaine et al 2002), they might represent an important galaxy evolution phase where a quasar nucleus is forming amid heavy dust obscuration (e.g., Sanders et al 1988). A population of mid-infrared-selected hyperluminous objects at $z>2$, which appear to be powered mainly by obscured active galactic nuclei (AGN), are emerging from Spitzer surveys (Houck et al 2005). As well as the origin of the enormous luminosity being of a great interest, examining the role of luminous counterparts of nearby Compton-thick Seyfert AGN to the X-ray background (XRB) and their detectability is also important, since the higher energy part of the XRB is not yet fully resolved (Worsley et al 2005).

IRAS F15307+3252 is one of the few classical IRAS-selected hyperluminous infrared galaxies at redshift of $z=0.926$ (Cutri et al 1994). With the currently popular cosmology with $H_{0}=70$ $\mathrm{km} \mathrm{s}^{-1} \mathrm{Mpc}^{-1}, \Omega_{\mathrm{M}}=0.27$, and $\Omega_{\Lambda}=0.73$, the luminosity distance is $6.1 \mathrm{Gpc}$ and and the bolometric luminosity is estimated to be $3 \times 10^{13} L_{\odot}$.

The large bolometric luminosity was once suspected to be the result of gravitational lensing (Liu, Graham \& Wright 1996) as in the case of IRAS F10214+4724 at $z=2.3$ (e.g., Broadhurst \& Lehar 1995). However Farrah et al (2002) have ruled this out based on HST imaging. Therefore, IRAS F15307+3252 indeed belongs among the most luminous objects in the universe.

The UV/optical emission-line spectrum of IRAS F15307+3252 is of Seyfert 2 type (Cutri et al 1994; Soifer et al 1994; Liu et al 1996; Evans et al 1998). Optical spectropolarimetry has revealed broad MgII $\lambda 2798$ emission and a rest-frame UV spectrum which is very similar in shape and intensity to that of typical quasars, indicating the presence of a dust-shrouded quasar nucleus (Hines et al 1995).

Despite the optical evidence for hidden active nuclei, hyperluminous infrared galaxies are generally very faint in X-ray (Wilman et al 1998; 2003; see Franceschini et al 2000; Iwasawa, Ettori \& 
Table 1. XMM-Newton observations of IRAS F15307+3252. Useful exposure times in unit of ks (and whole duration of exposure including the high background intervals in parenthesis) for the respective EPIC cameras.

$\begin{array}{lcc}\text { Date } & \begin{array}{c}\text { pn } \\ \text { ks }\end{array} & \begin{array}{c}\text { MOS } \\ \text { ks }\end{array} \\ & & \\ \text { 2002 Jul 30 } & 6.7(24) & 10(25) \\ \text { 2004 Jul 29 } & 9.6(30) & 12(31) \\ \text { 2004 Dec 30 } & 5.1(23) & 11(18)\end{array}$

Fabian 2000 for IRAS P09104+4101; Iwasawa 2001; Alexander et al 2005 for IRAS F10214+4724). IRAS F15307+3252 is no exception and previous X-ray observations with ROSAT and ASCA failed to detect any X-rays (Fabian et al 1996; Ogasaka et al 1997). We report here the detection of faint X-ray emission from this hyperluminous infrared galaxy and its properties obtained from XMM-Newton observations, and discuss their implications.

\section{OBSERVATIONS}

IRAS F15307+3252 was observed with the EPIC cameras of XMM-Newton on three occasions (Table 1). Both EPIC pn and MOS cameras operated in the full-window mode with the medium filter in all the three observations. A significant fraction of the datasets are affected by high radiation background. A larger fraction of the observed intervals are affected in the pn data than in the MOS data. Time intervals of quiescent background (count rate less than $0.3 \mathrm{ct} \mathrm{s}^{-1}$ for each of the MOS and less than $1.2-1.5 \mathrm{ct} \mathrm{s}^{-1}$ for the pn) were selected, based on the single event light curves at energies above $10 \mathrm{keV}$ taken from the whole field of each detector. The useful exposures from the three observations are 6, 10, and $5 \mathrm{ks}(21$ $\mathrm{ks}$ in total) for the pn camera, and 10,12, and $11 \mathrm{ks}$ (33 ks in total) for the MOS cameras. Single and double events recorded by the detectors were used for the data analysis presented here. The data reduction was performed by the standard XMM-Newton software package SAS 6.1 and LHEASOFT 5.3.1.

\section{RESULTS}

\subsection{Photometric study}

IRAS F15307+3252 is detected with XMM-Newton but is very faint, particularly at energies above $2 \mathrm{keV}$. With so few detected counts, the individual observations do not warrant an independent analysis. We integrated all three datasets to examine source detection first in different energy bands. Compton-thick obscuration can be a plausible explanation for the X-ray faintness of IRAS F15307+3252. Since a strong Fe K $\alpha$ feature at rest-frame 6-7 keV would characterise the X-ray spectrum in such a case, we designed a photometric study by dividing the whole bandpass into the following four bands: $0.5-2 \mathrm{keV}, 2-3 \mathrm{keV}, 3-4 \mathrm{keV}$, and 5-10 keV, so that $\mathrm{Fe} \mathrm{K} \alpha$ feature falls in the $3-4 \mathrm{keV}$ band by the galaxy redshift $(z=0.926)$. X-ray images in the four bands were constructed by adding all the pn and MOS data from the three observations, which are shown in Fig. 1.

IRAS F15307+3252 is detected at more than $3 \sigma$ in all but the 2-3 keV band images (see Table 1). The source is strongly detected at lower energies below $2 \mathrm{keV}$ for which further details are presented below. This component does not seem to stretch well above $2 \mathrm{keV}$, as suggested by the lack of detection in the $2-3 \mathrm{keV}$ band.

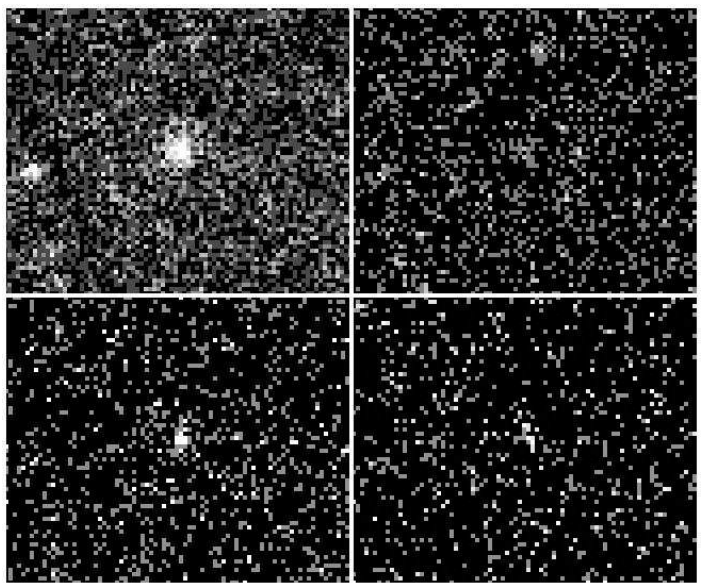

Figure 1. The X-ray images in the four energy bands: clockwise from upper-left: $0.5-2 \mathrm{keV} ; 2-3 \mathrm{keV}$; 5-10 keV; and 3-4 keV. The images were constructed by adding all the pn and MOS detectors together from all the three observations. The size of each image is $5^{\prime} \times 4^{\prime} .5$. The backgroundcorrected counts in the respective bands and their signal to noise ratios (in parenthesis) are 0.5-2 keV: 186 ct $(12 \sigma) ; \mathbf{2}-\mathbf{3} \mathbf{~ k e V : ~} 11$ ct $(2 \sigma) ; \mathbf{3}-\mathbf{4} \mathbf{~ k e V : ~}$ 34 ct $(5 \sigma)$; and 5-10 keV: 33 ct $(4 \sigma)$.

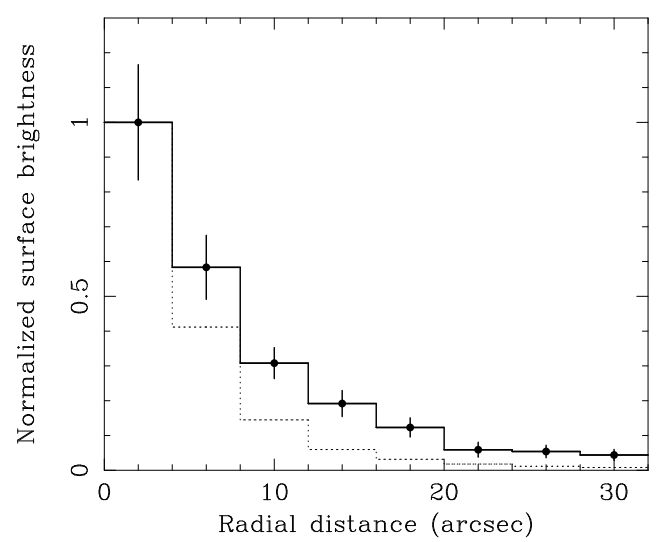

Figure 2. The radial X-ray surface brightness profiles of the $0.5-2 \mathrm{keV}$ emission from IRAS F15307+3252 (solid-line histogram) as observed with XMM-Newton, together with that from NGC4395 (dotted-line histogram) in the same band. The profiles have been normalized at the innermost bin. NGC4395 is a point-like source thus represents the point spread function.

Note, however, a remarkable recovery of the source in the 3-4 keV band. This indicates a strong excess in the $3-4 \mathrm{keV}$ range, where $\mathrm{Fe}$ $\mathrm{K} \alpha$ emission is expected. The $2-3 \mathrm{keV}$ drop-out and the marginal detection in the 5-10 keV band suggest a rather hard underlying continuum in the $2-10 \mathrm{keV}$ (the rest frame $4-20 \mathrm{keV}$ ) range. These are characteristics of the X-ray spectrum of a heavily absorbed active nucleus.

\subsection{Extended soft $\mathrm{X}$-ray emission and its origin}

The soft X-ray emission below $2 \mathrm{keV}$ found at the position of IRAS F15307+3252 is not point-like. The radial profile of the soft band source is compared with that of the nuclear source in NGC4395, which was also observed with the XMM-Newton in the full-window mode (Fig. 2). Since the X-ray emission from NGC4395 has been found to be point-like as viewed by the Chandra ACIS-S (Moran et al 2005), it represents the point spread func- 

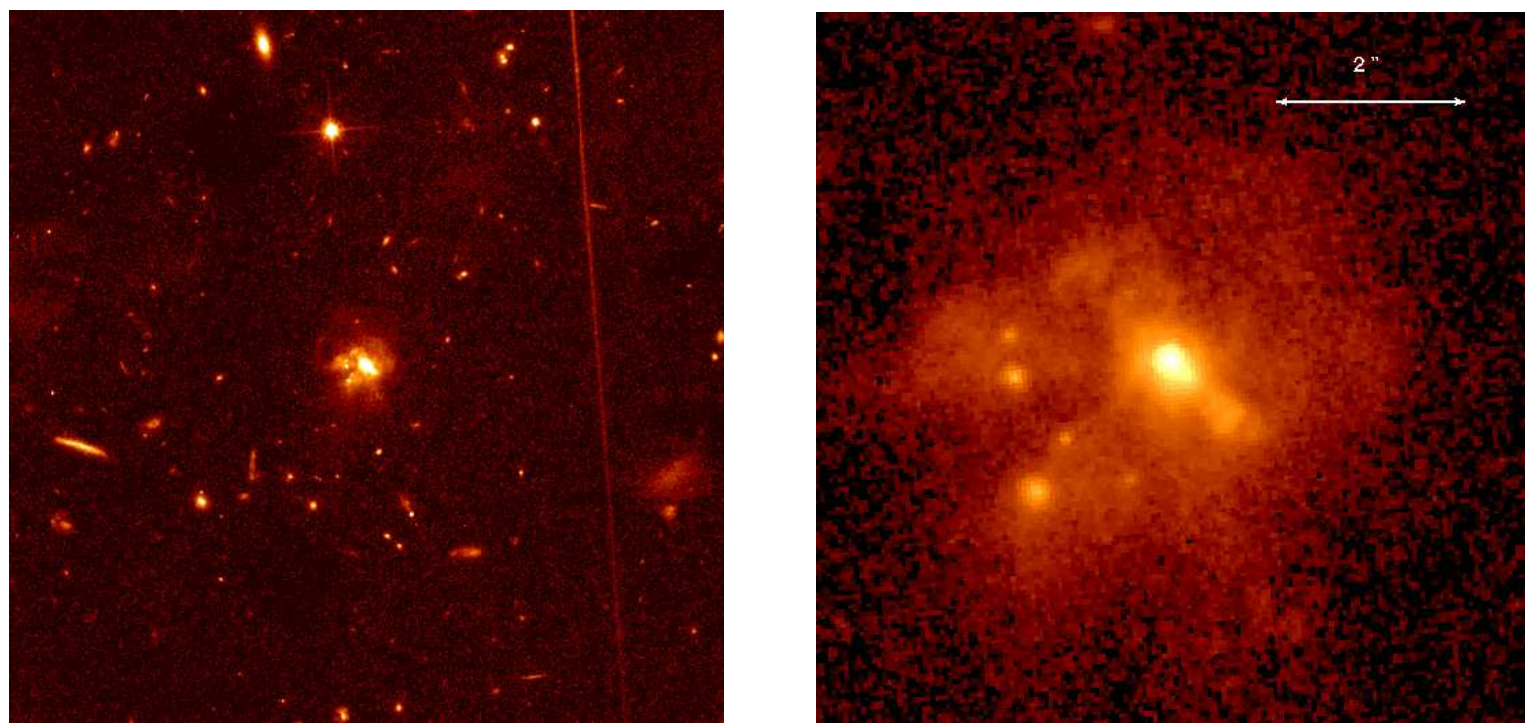

Figure 3. The HST/ACS images of the $1^{\prime} \times 1^{\prime}$ region (left) around IRAS F15307+3252 (right), taken through the F814W filter (rest-frame blue). North is up and East to the left. The images have been gaussian-smoothed by 1 pixel $\left(\simeq 0^{\prime \prime} .05\right)$. The large-scale image shows a chain of small galaxies $11^{\prime \prime}-15^{\prime \prime}(\sim 100$ $\mathrm{kpc}$ at $z=0.93$ ) to the south of the IRAS galaxy, which is reminiscent to Markarian's chain of galaxies in the Virgo cluster lying at $\sim 300 \mathrm{kpc}$ in projected distance from M87. The zoom-up of IRAS F15307+3252 shows complex structures within the diffuse envelope, including several knots, a cavity of a conical shape with a sharp boundary to the south-east, and arc-like structures to the north-east.

tion (PSF). Although adding different detectors and observations together might have resulted in a blurred PSF at some degree, a comparison of the radial profile with other point sources in the field confirms the extended nature of the soft X-ray emission from IRAS F15307+3252.

The angular scale at $z=0.926$ with the adopted cosmology is $\simeq 8 \mathrm{kpc} \operatorname{arcsec}^{-1}$. Detection of a source size broader than the PSF (FWHM $\simeq 5-6$ arcsec) means that the source is extending at least $\sim 50 \mathrm{kpc}$. Fitting a Lorentzian function to the respective radial profiles of IRAS F15307+3252 and NGC4395 implies that the FWHM of the intrinsic source extension is about $21 \pm 5$ arcsec, corresponding to $85 \mathrm{kpc}$ in radius. This is larger than the effective radius ( $\sim 12 \mathrm{kpc}$, Farrah et al 2002) of the giant elliptical host of IRAS F15307+3252, of which the surface brightness distribution is described well with the de Vaucouleur's profile (see also Liu et al 1996).

No galaxy cluster has so far been reported for the region around IRAS F15307+3252. However, IRAS F15307+3252 is a luminous $\left(M_{I}=-26.4\right.$, Farrah et al 2002) giant elliptical, inside which some substructures have been found, indicating galaxy mergers or interaction (Fig. 3, also Soifer et al 1994; Liu et al 1996; Farrah et al 2002). The galaxy is $\sim 1.5$ mag more luminous than the Virgo brightest galaxy M87, and should be in a relatively massive dark matter halo. Fig. 3 shows the HST/ACS image of the region around IRAS F15307+3252, retrieved from the HST archive at STScI. The data were taken on 2002 August 18 in a 2,120-s exposure, using the F814W filter (approximately $I$ band). While there is no clear evidence for a galaxy concentration centred on the IRAS galaxy as such expected in a rich cluster, a chain of several small galaxies $11-15 \operatorname{arcsec}(\sim 100 \mathrm{kpc})$ to the south is seen, which lies within the X-ray extension, indicating a moderate galaxy overdensity. The HST image also shows that both IRAS F15307+3252 and the several nearby compact knots (which Farrah et al 2002 ascribe to the remnants of merging companions) are embedded in an envelope of diffuse emission out to a radius of $\sim 3$ arcsec. There is considerable structure within this emission, including a sharp conical-shaped cavity to the SE with its apex at the AGN. Further arc-shaped structures concentric to the active nucleus and perpendicular to the cavity are also apparent, which could be due to $[\mathrm{OII}] \lambda 3727$ located near the edge of the filter bandpass, representing ionization cones.

Fitting jointly the pn and MOS spectra (Fig. 4) gives a temperature of $k T=2.1_{-0.4}^{+0.6} \mathrm{keV}$ (hereafter, the errors for spectral parameters are of the 90 per cent confidence region for one parameter of interest). The $0.4-2.8 \mathrm{keV}$ band was fitted with half solar metallicity and Galactic absorption of $N_{\mathrm{H}}=2 \times 10^{20} \mathrm{~cm}^{-2}$ (Dickey \& Lockman 1990) being assumed. The observed 0.5-2 keV flux is $1.2 \times 10^{-14} \mathrm{erg} \mathrm{cm}^{-2} \mathrm{~s}^{-1}$. The absorption-corrected bolometric luminosity is estimated to be $\sim 1 \times 10^{44} \mathrm{erg} \mathrm{s}^{-1}$. The temperature and bolometric luminosity are on the $L-T_{\mathrm{X}}$ relation for galaxy clusters, groups and elliptical galaxies (e.g., Fukazawa et al 2004), and IRAS $\mathrm{F} 15307+3252$ lies in the region for a poor cluster (it is comparable to the Virgo cluster in temperature and luminosity). We tentatively identify the extended soft X-ray emission with hot gas associated with a relatively poor cluster around the IRAS galaxy.

Whilst soft X-ray emission in some hyperluminous infrared galaxies has been ascribed to star formation (Wilman et al 2003; Alexander et al 2005), the large source size, high temperature and large soft X-ray to infrared luminosity ratio suggest that a starburst is unlikely to be a major component of the soft X-ray emission in IRAS F15307+3252 (see also Section 4.2). The cluster emission has a relatively short radiative cooling time of $\leqslant 4 \mathrm{Gyr}$. This would lead to a cooling flow of $\sim 150 M_{\odot} \mathrm{yr}^{-1}$ if there is no heating to balance cooling (there is a 6 mJy FIRST radio source at $1.4 \mathrm{GHz}$; Becker, White \& Helfand 1995).

\subsection{Fe K line and hard X-ray emission}

The photometric study (Section 3.1) demonstrated that X-ray emission of IRAS F15307+3252 shows a strong excess in the 3-4 keV range, which is probably due to a redshifted $\mathrm{Fe} \mathrm{K} \alpha$ line. There is a very faint underlying continuum, which extends to higher energies. 


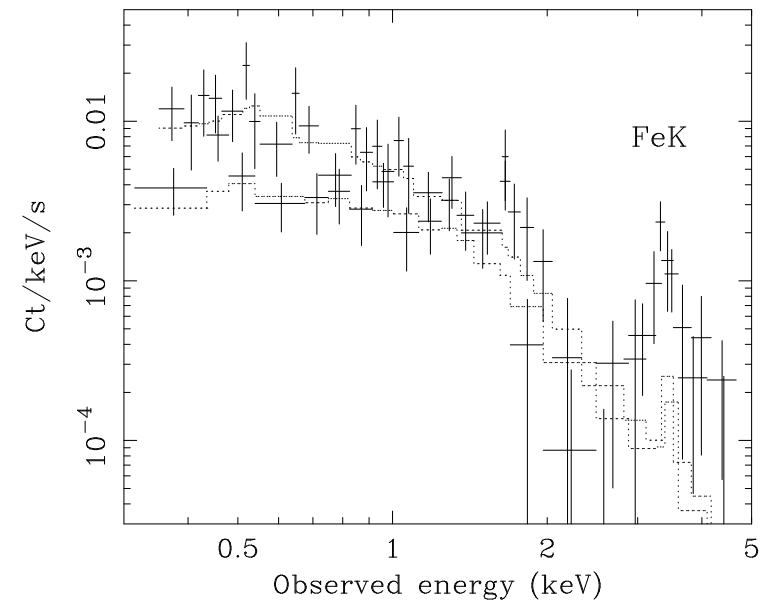

Figure 4. The co-added pn and MOS spectra of IRAS F15307+3252. The energy scale is as observed. A strong excess feature in the 3-4 keV range can be identified with $\mathrm{Fe} \mathrm{K} \alpha$. The dotted-line histograms are the thermal emission spectrum (MEKAL) with $k T=2.1 \mathrm{keV}$, half solar metallicity and the Galactic absorption, best-fitting the $0.4-3 \mathrm{keV}$ data. Note that the extrapolation of the thermal emission spectrum to higher energy clearly underestimates the observed Fe K emission flux, which is therefore of the different origin.

The faint $5-10 \mathrm{keV}$ band is too noisy for a spectral study and only fit for a photometric measurement. The spectral data in the 2-5 $\mathrm{keV}$ band can be fitted with a narrow gaussian with a flat powerlaw $(\Gamma<0.8)$. The centroid of the gaussian is $6.47_{-0.11}^{+0.10} \mathrm{keV}$ in the rest frame. The fit shows a line width of $0.1 \mathrm{keV}$ in gaussian dispersion, but the data also agree with no broadening. Assuming this feature is all due to line emission yields a line flux of $1.3_{-0.6}^{+0.5} \times 10^{-6}$ $\mathrm{ph} \mathrm{s}^{-1} \mathrm{~cm}^{-2}$. Since the continuum is too faint to obtain a reasonable constraint, the equivalent width (EW) of the line is accordingly uncertain, but it is likely to be larger than $2 \mathrm{keV}$.

The observed 2-5 keV flux is $1 \times 10^{-14} \mathrm{erg} \mathrm{cm}^{-2} \mathrm{~s}^{-1}$ (corresponding to a $4-10 \mathrm{keV}$ rest frame luminosity of $\simeq 4 \times$ $10^{43} \mathrm{erg} \mathrm{s}^{-1}$ ), most of which originates from the line feature. Extrapolating the $2-5 \mathrm{keV}$ continuum model with $\Gamma=-0.5$ predicts source counts which are comparable to the detected counts in the 5$10 \mathrm{keV}$ image at the given exposure times. The observed 5-10 keV flux derived from the extrapolation is $2.5 \times 10^{-14} \mathrm{erg} \mathrm{cm}^{-2} \mathrm{~s}^{-1}$, and the rest-frame $10-20 \mathrm{keV}$ luminosity estimated from the flux is $1 \times 10^{44} \mathrm{erg} \mathrm{s}^{-1}$.

\section{DISCUSSION}

\subsection{The hidden quasar nucleus}

The detection of the prominent $\mathrm{Fe} \mathrm{K} \alpha$ line strongly indicates the presence of a Compton-thick AGN in IRAS F15307+3252. The large $\mathrm{EW}$ of the $\mathrm{Fe} \mathrm{K} \alpha$ and the deficit of X-ray emission just below the line imply the lack of the direct continuum emission in the $\mathrm{Fe} \mathrm{K}$ band, which means the absorbing column density to be $N_{\mathrm{H}} \sim 1 \times 10^{24} \mathrm{~cm}^{-2}$ or larger. Thus, together with the line centroid at $6.4 \mathrm{keV}$, the hard X-ray emission from IRAS F15307+3252 is dominated by reflection.

Using the observed line luminosity, we made a rough estimate of the minimum rest-frame $2-10 \mathrm{keV}$ luminosity which is required to produce the observed $\mathrm{Fe} \mathrm{K}$ emission. Assumptions made for this calculation are as following: $\mathrm{Fe} \mathrm{K} \alpha$ emission is produced in cold matter with Solar abundance $\left(N_{\mathrm{Fe}} / N_{\mathrm{H}}=3.3 \times 10^{-5}\right.$, Morrison \& McCammon 1983), irradiated by a power-law source with a typical quasar photon-index of $\Gamma=2$; the continuum photons above the $\mathrm{K}$ shell ionization threshold energy $(7.11 \mathrm{keV})$ are converted into $\mathrm{Fe}$ $\mathrm{K} \alpha$ at $6.4 \mathrm{keV}$ with the efficiency calculated by Basko (1978) and George \& Fabian (1991), as such that would produce $E W=150 \mathrm{eV}$, if an observer see both the illuminating source and reflection from the cold slab behind it; and the solid angle of the cold matter viewed by the illuminating source is $4 \pi$. With these assumptions, the ratio $L_{\mathrm{FeK}} / L_{2-10}$ is found to be about 3 per cent. Since not all the reflecting surfaces are likely visible to an observer, the minimum 2 $10 \mathrm{keV}$ luminosity to produce the observed $\mathrm{Fe} \mathrm{K}$ line is estimated to be $L_{2-10}=(0.03)^{-1} \times 4 \times 10^{43} \sim 1 \times 10^{45} \mathrm{erg} \mathrm{s}^{-1}$. The ratio of bolometric to the $2-10 \mathrm{keV}$ band luminosity, $f_{\mathrm{Xbol}}=L_{\mathrm{bol}} / L_{2-10}$, is typically $30-50$ for quasars (Elvis et al 1994). With this bolometric correction, the bolometric luminosity of the AGN in IRAS $\mathrm{F} 15307+3252$ is found to be $L_{\mathrm{bol}}^{\mathrm{AGN}} \geqslant 3 \times 10^{46} f_{\mathrm{Xbol}, 30} \mathrm{erg} \mathrm{s}^{-1}$, where $f_{\mathrm{Xbol}}=30 f_{\mathrm{Xbol}, 30}$. Therefore a large fraction of the bolometric luminosity $\left(10^{47} \mathrm{erg} \mathrm{s}^{-1}\right)$ is likely to be powered by the hidden AGN, consistent with previous estimates (e.g., Yun \& Scoville 1998; Aussel et al 1998; Verma et al 2001; Peeters, Spoon \& Tielens 2004).

The reflected X-ray emission originates from optically thick matter and is of a distinct origin from the scattered UV/optical light revealed by spectropolarimetry (Hines et al 1995). The large polarization in the UV/optical bands (16-20 per cent) is presumably due to dust scattering.

\subsection{Black holes in hidden quasars and their environment}

The black hole masss hosted in IRAS F15307+3252 is estimated to be $M_{\mathrm{BH}} \sim 1.3 \times 10^{9} M_{\odot}$, using the empirical relation based on the virial theorem for the BLR obtained by McLure \& Jarvis (2002) with the MgII line width of FWHM $\sim 10,000 \mathrm{~km} \mathrm{~s}^{-1}$ and the 3000 Å luminosity $\left(\lambda L_{\lambda} \approx 2 \times 10^{45} \mathrm{erg} \mathrm{s}^{-1}\right)$ of the intrinsic UV spectrum derived from the spectropolarimetry (Hines et al 1995). The bolometric luminosity of this object is then about half the Eddington luminosity for the estimated black hole mass.

IRAS F15307+3252 shares many properties with another hyperluminous infrared galaxy IRAS $09104+4109$. In their spectral energy distribution, the infrared to optical and $60 \mu \mathrm{m}$ to $1.5 \mathrm{GHz}$ luminosity ratios for both galaxies are around 300 and 50, respectively (Cutri et al 1994). Both contain a hidden quasar revealed by optical spectropolarimetry (Hines et al 1999), which appears to dominate the energetics of the infrared emission, and their Xray spectra show characteristics of Compton-thick AGN (Franceschini et al 2000; Iwasawa et al 2001). The black hole mass of IRAS 09104+4109 estimated by the same technique as described above is $3 \times 10^{9} M_{\odot}$ (V.D. Ivanov, priv comm), similar to that of IRAS F15307+3252. These suggest that both galaxies have a well-grown black hole emitting at a high efficiency, although their radiation is strongly attenuated by dust shrouds in the optical wavelength and by cold gas with a large Thomson depth $\left(\tau_{\mathrm{T}} \geqslant 1\right)$ in the line-of-sight in X-ray, respectively. Non-detection of CO (Evans et al 1998; Yun $\&$ Scoville 1998) in either galaxies limits the material needed for a vigorous star formation. The deficit of cold $(T \sim 40 \mathrm{~K})$ dust in both galaxies inferred by the non-detection with SCUBA (Deane \& Trentham 2001; which places the limit of the contribution of cold dust to the bolometric luminosity less than 0.3 per cent) can be a natural consequence of the absence of a strong starburst. Perhaps in these two galaxies, a mature black hole is still efficiently accreting material from small radii, while star formation on a large scale 
has already been terminated. As Hines et al (1995) suggested, the AGNs in these galaxies are viewed from an unfavourable direction but otherwise are indistinguishable from normal quasars.

One remaining similarity between the two objects is their environment. IRAS $09104+4109$ is a giant elliptical located at the centre of a rich cluster at $z=0.44$ (Kleinmann et al 1988), which is one of the most luminous X-ray clusters with $L_{\mathrm{bol}}^{\mathrm{CL}} \simeq$ $3 \times 10^{45} \mathrm{erg} \mathrm{s}^{-1}$ (Fabian \& Crawford 1995; Iwasawa et al 2001). Despite the much lower luminosity and higher redshift, IRAS F15307+3252 appears to be in a cluster as the extended X-ray emission indicates. If this is the case, galaxy mergers in an overdensity region may be a necessary condition for making a luminous quasar. This contrasts with nearby lower luminosity infrared galaxies which are not usually found in a rich environment (Sanders \& Mirabel 1996). On the other hand, quasars (Wold et al 2000, 2001) and possibly hyperluminous infrared galaxies (Farrah et al 2004) tend to reside in a moderately dense environment, and which is in agreement with galaxy and quasar formation model based on a hierarchical assembly scenario (e.g., Enoki, Nagashima \& Gouda 2003). This has implications for a search for Type II quasars in Xray. In an unobscured quasar, the nuclear emission dominates the total X-ray emission even if there is some emission from a cluster with which the quasar is associated. However, in an obscured quasar, the nuclear emission is suppressed and soft X-ray emission from a putative cluster could be a main X-ray source rather than the quasar itself. Whether faint reflected light of a hidden nucleus is detectable depends on its luminosity. Since such a component was only barely detected in IRAS F15307+3252, one of the most luminous galaxies, with a $30 \mathrm{ks}$ XMM-Newton exposure, there are clear difficulties in detecting Compton-thick AGN at redshift of $z \geqslant 1$.

\section{ACKNOWLEDGEMENTS}

This paper is based on observations obtained with XMM-Newton, an ESA science mission with instruments and contributions directly funded by ESA Member States and NASA. ACF and CSC thank the Royal Society for support. KI thanks PPARC for support.

\section{REFERENCES}

Alexander D.M., Chartas G., Bauer F.E., Brandt W.N., Simpson C., Vignali C., 2005, MNRAS, in press

Aussel H., Gerin M., Boulanger F., Desert F. X., Casoli F., Cutri R. M., Signore M., 1998, A\&A, 334, L73

Basko M.M., 1978, ApJ, 223, 268

Becker R.H., White R.L., Helfand D.J., 1995, ApJ, 450, 559

Broadhurst T., Lehar J., 1995, ApJ, 450, L41

Cutri R.M., Huchra J.P., Low F.J., Brown R.L., van den Bout P.A., 1994, ApJ, 424, L65

Deane J. R., Trentham N., 2001, MNRAS, 326, 1467

Dickey J. M., Lockman F. J., 1990, ARA\&A, 28, 215

Elvis M., et al, 1994, ApJS, 95, 1

Enoki M., Nagashima M., Gouda N., 2003, PASJ, 55, 133

Evans A. S., Sanders D. B., Cutri R. M., Radford S. J. E., Surace J. A., Solomon P. M., Downes D., Kramer C., 1998, ApJ, 506, 205

Fabian A.C., Crawford C.S., 1995, MNRAS, 274, L63

Fabian A. C., Cutri R. M., Smith H. E., Crawford C. S., Brandt W. N., 1996, MNRAS, 283, L95

Farrah D., Verma A., Oliver S., Rowan-Robinson M., McMahon R., 2002, MNRAS, 329, 605

Farrah D., Geach J., Fox M., Serjeant S., Oliver S., Verma A., Kaviani A., Rowan-Robinson M., 2004, MNRAS, 349, 518
Franceschini A., Bassani L., Cappi M., Granato G. L., Malaguti G., Palazzi E., Persic M., 2000, A\&A, 353, 910

Fukazawa Y., Makishima K., Ohashi T., 2004, PASJ, 56, 965

George I.M., Fabian A.C., 1991, MNRAS, 249, 352

Hines D. C., Schmidt G. D., Smith P. S., Cutri R. M., Low F. J., 1995, ApJ, 450, L1

Houck J. R., et al., 2005, ApJ, 622, L105

Iwasawa K., 2001, AIP Conf. Proc. 599,: X-ray Astronomy: Stellar Endpoints, AGN, and the Diffuse X-ray Background, 599, 169

Iwasawa K., Fabian A. C., Ettori S., 2001, MNRAS, 321, L15

Kleinmann S. G., Hamilton D., Keel W. C., Wynn-Williams C. G., Eales S. A., Becklin E. E., Kuntz K. D., 1988, ApJ, 328, 161

Liu M. C., Graham J. R., Wright G. S., 1996, ApJ, 470, 771

McLure R. J., Jarvis M. J., 2002, MNRAS, 337, 109

Moran E., Eracleous M., Leighly K.M., Chartas G., Filippenko A.V., Ho L.C., Blanco P.R., 2005, AJ, 129, 2108

Morrison R., McCammon D., 1983, ApJ, 270, 119

Ogasaka Y., Inoue H., Brandt W. N., Fabian A. C., Kii T., Nakagawa T., Fujimoto R., Otani C., 1997, PASJ, 49, 179

Peeters E., Spoon H. W. W., Tielens A. G. G. M., 2004, ApJ, 613, 986

Rowan-Robinson M., 2000, MNRAS, 316, 885

Sanders D.B., Mirabel F.I., 1996, ARAA, 34, 749

Sanders D. B., Soifer B. T., Elias J. H., Madore B. F., Matthews K., Neugebauer G., Scoville N. Z., 1988, ApJ, 325, 74

Stanford S.A., Stern D., van Breugel W., De Breuck C., 2000, ApJS, 131, 185

Soifer B. T., Neugebauer G., Matthews K., Armus L., 1994, ApJ, 433, L69

Tremaine S.D., et al, 2002, ApJ, 574, 740

Verma A., Rowan-Robinson M., McMahon R., Andreas Efstathiou A. E., 2002, MNRAS, 335, 574

Wilman R. J., Fabian A. C., Crawford C. S., Cutri R. M., 2003, MNRAS, 338, L19

Wilman R. J., Fabian A. C., Cutri R. M., Crawford C. S., Brandt W. N., 1998, MNRAS, 300, L7

Wold M., Lacy M., Lilje P. B., Serjeant S., 2001, MNRAS, 323, 231

Wold M., Lacy M., Lilje P. B., Serjeant S., 2000, MNRAS, 316, 267

Worsley M. A., et al., 2005, MNRAS, 357, 1281

Yun M. S., Scoville N. Z., 1998, ApJ, 507, 774 